\title{
Direct immunofluorescent test for the detection of gonorrhoea
}

\author{
J. KLANICA AND M. STEJSKALOVÁ \\ From the Hygiene Station of the National Committee of the City of Prague, Department of Microbiology, \\ and the National Health Centre, Prague 3, Gynaecological Department, Czechoslovakia
}

\section{Summary}

In the course of 4 years we have examined a total of 1,100 women with chronic gynaecological complaints. The direct fluorescent antibody test was used successfully for the detection of gonorrhoea. In Group 1 (220 patients) we found $39 \cdot 1$ per cent. with gonorrhoea. In group 2 (220 patients) the percentage fell to 27.7 per cent., in Group 3 (220 patients) to 23.2 per cent., in Group 4 (220 patients) to 12.3 per cent., and in Group 5 (220 patients) to 11.8 per cent. By employing a consistent programme of diagnosis and therapy we succeeded in reducing the incidence of gonorrhoea in the Prague 3 District from 39.1 per cent. in 1970 to 11.8 per cent. in 1973-74.

In all we discovered 251 female patients suffering from gonorrhoea, who would otherwise have escaped observation and registration. When patients are not registered as sources of gonococcal infection, special cultures are not performed as a routine, and these women would therefore infect the same number of men at least. The immunofluorescent method proved to be of great value. The direct FAT is a superior test; even in cases in which, because of damage by such factors as antibiotics, Neisseria gonorrhoeae does not grow on artificial media, gonorrhoea can be detected by this method.

\section{Introduction}

Brown (1968) stated that the chief reservoir of gonorrhoea infection is probably the female without a marked vaginal discharge. This paper is based on the assumption that latent sources of gonorrhoea frequently exist even in the present era of mass application of antibiotics. Female patients may be treated for another infection with doses of antibiotics insufficient to cure gonorrhoea. In these circumstances latent infection persists and may later be activated

Received for publication April 22, 1975 by some physiological or pathological process (Palouš, Sturma, Křeček, Potužník, and Hausner, 1971). It is suggested that gonorrhoea may be present in women with persistent vaginal discharge resistant to conventional therapy. In February, 1970, we started a long-term investigation of female patients with chronic urogenital conditions who were attending the gynaecological department in Prague 3. Our first results have already been published (Klanica and Stejskalová, 1972). For diagnostic purposes we concentrated on the immunofluorescent method (fluorescent antibody test (FAT)) which (according to Fry and Wilkinson, 1964; Gallwey, Nicol, and Ridley, 1967; Henderson, Rutherford, Phelps, and Robertson, 1970; Thin, 1970; Thin, Williams, and Nicol, 1971) is more sensitive than cultures. The direct or immediate FAT was used as previously described (Klanica and Stejskalová, 1972)

\section{Material and methods}

Between February, 1970, and March, 1974, a series of 1,100 female patients suffering from chronic conditions of the genito-urinary system was investigated. Most of them had been repeatedly treated unsuccessfully with a number of drugs such as metronidazole, penicillin, chloramphenicol, tetracycline, and streptomycin.

SPECIMENS

Smears for direct FAT were taken with a special wire loop from the cervix, urethra, and rectum and inoculated on to blood agar for general bacteriological flora, and on MKALC medium (a modified blood agar with the addition of lincomycin and colistin) for gonococci. After inoculation the blood agar was incubated at $37^{\circ} \mathrm{C}$. for $24 \mathrm{hrs}$, and the MKALC medium was incubated at an increased $\mathrm{CO}_{2}$ pressure for $48 \mathrm{hrs}$. $N$. gonorrhoeae was identified by the oxidase reaction, by microscopical examination of suspected colonies, and by sugar fermentation tests. An antibiogram was also performed for $N$. gonorrhoeae. The MKALC medium and the fermentation media were prepared according to the instructions of the Czechoslovak National Reference Laboratory for Gonorrhoea. When the FAT gave positive results at the first examination but the culture was negative, three further sets of cultures were taken within the next month. 


\section{CONJUGATE AND STAINING TECHNIQUE}

The conjugate BACTo-FA $N$. gonorrhoeae (Difco) was used according to the manufacturers' instructions. Smears of secretions were dried in air, fixed for $5 \mathrm{~min}$. in methyl alcohol, and again left to dry. They were then coated with the conjugate and incubated in a moist chamber for $1 \mathrm{hr}$ at $37^{\circ} \mathrm{C}$. The slides were then counter-stained with Evan's blue $(1: 100,000)$ for $5 \mathrm{~min}$. after twice washing with phosphate buffered saline (PBS) at $\mathrm{pH} 7 \cdot 2$ and with distilled water. Finally the smears were washed again with PBS and distilled water.

\section{OPTICAL EQUIPMENT}

Smears were read with a light microscope of Russian make M L-2. The light source in this microscope is a mercury vapour arc lamp DRŽ-250; a combination of excitation filters corresponding to Wratten No. 18A and a barrier corresponding to Wratten No. 61 were used. The slides were examined by annular incident illumination with an oil immersion $\times 90$ objective, a binocular head $\times 1$, and oculars $\times 4$

\section{CONTROLS}

Each smear was paired with a control on a different slide. Stained smears of a known culture of $N$. gonorrhoeae were used as positive controls and stained smears of BACTOCloacae Desiccated served as negative controls. Typical intracellular diplococci fluoresced brightly and, if there was an identical fluorescence in the positive controls, these were assessed as positive findings. Slides with no specific fluorescence were considered to be negative.

\section{Results}

The series of 1,100 females was divided into five groups of 220 patients each, arranged chronologically from February, 1970, to March, 1974. These provide a survey of the changing situation in the incidence of gonorrhoea in Prague 3. The results are given in the Table.

\section{Group 1}

In the 220 patients examined between February, 1970, and October, 1970, a positive FAT result was seen in 114 patients, but at the first examination there were only 49 positive cultures $(22.3$ per cent.). There was one patient with a positive culture and negative FAT. Three subsequent examinations produced 37 more positive cultures ( 16.8 per cent.). The FAT results were not confirmed by culture in 28 cases and therefore no definite diagnosis was made.

With only one set of cultures from urethra, cervix, and rectum by the routine procedure, 37 out of a total of 86 cases of gonorrhoea would have been missed.

\section{Group 2}

In the 220 patients from October, 1970, to March, 1971,61 cases of gonorrhoea were detected, 36 after the first culture and 25 after repeated cultures following a positive FAT. In nineteen cases in which the FAT was positive, the cultures were negative. There

TABLE Detection of N. gonorrhoeae in five groups of 220 women with chronic genito-urinary conditions

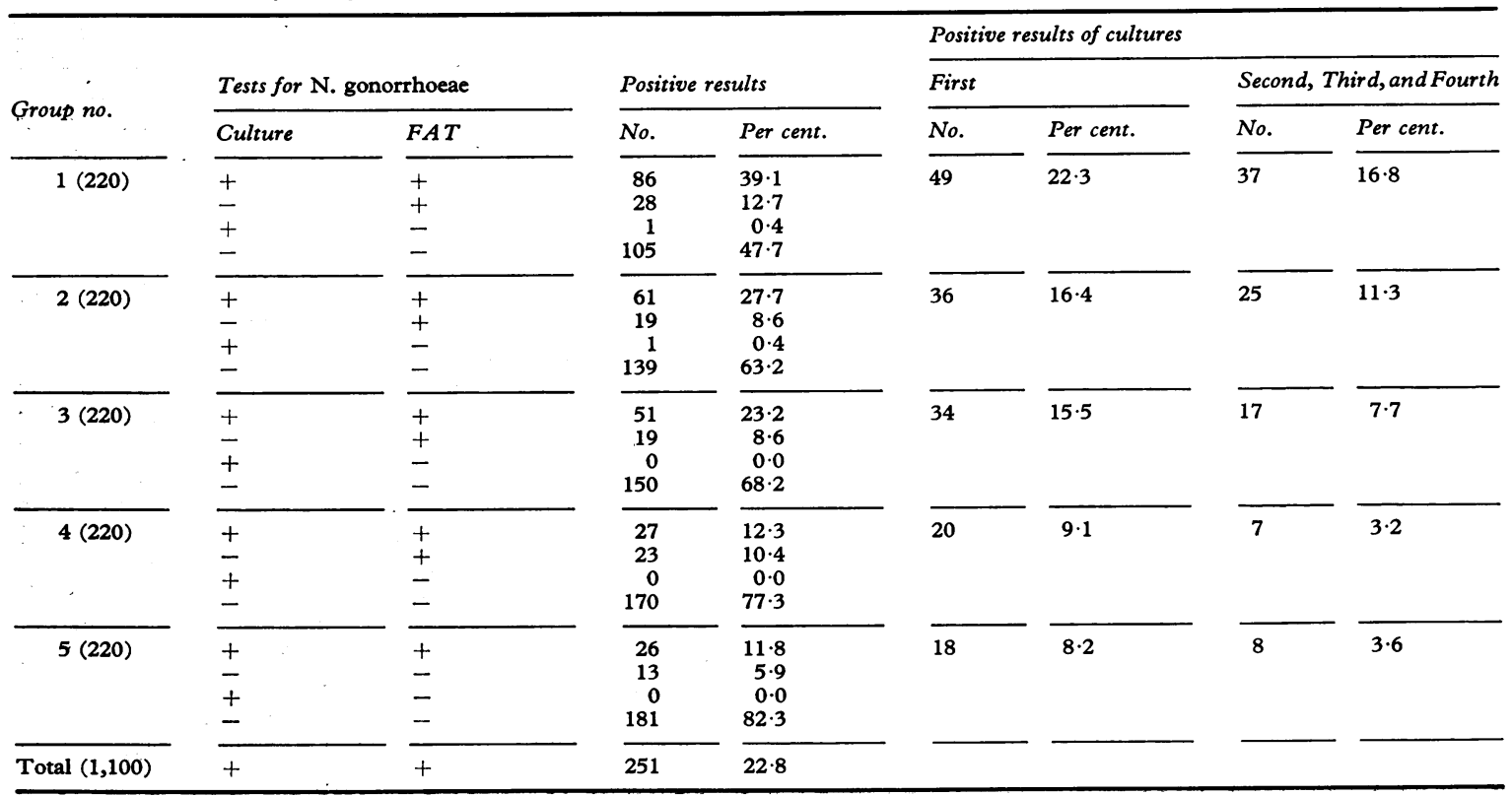


was one case with a positive culture and a negative FAT.

\section{Group 3}

In the 220 patients from March, 1971, to March, 1972, 51 cases of gonorrhoea were diagnosed, 34 immediately after the first culture and seventeen after repeated cultures. In nineteen cases in which the FAT was positive the cultures were negative.

\section{Group 4}

In 220 patients from March, 1972, to April, 1973, 27 cases of gonorrhoea were diagnosed, twenty after the first culture and seven after repeated cultures. In 23 cases in which the FAT was positive the cultures were negative.

\section{Group 5}

In 220 patients from April, 1973, to March, 1974, 26 cases of gonorrhoea were detected, eighteen after the first culture and eight after repeated cultures. Thirteen cases in which the FAT was positive had negative cultures.

The percentage of cases of gonorrhoea therefore fell from 39.1 to 11.8 per cent. during the four years, and 251 cases of gonorrhoea $(22.8$ per cent.) were detected in the 1,100 women with chronic inflammation of the genito-urinary tract.

\section{Discussion}

Many attempts have been made to apply the immunofluorescent method to the detection of $N$. gonorrhoeae. Deacon, Peacock, Freeman, and Harris (1959) first referred to this method. Kellogg and Deacon (1964), Danielsson (1965), and Sommerville (1968), elaborated rapid immunofluorescent staining techniques. Modern conjugates are so sensitive and specific that cross-reactions with streptococci can be almost excluded (Lind, 1967; Lind and Rhodeus, 1970).

The higher detection rate of gonorrhoea with the FAT compared with traditional cultures has been reported by Fry and Wilkinson (1964), Gallwey and others (1967), Lucas, Price, Thayer, and Schroeter (1967), Henderson and others (1970), Thin (1970), Thin and others (1971), and Enfors, Eriksson, Kaaman, and Krogh (1973). In the present study the FAT again proved to be more sensitive than culture.

The high rate (39.1 per cent.) of gonorrhoea in our first group was surprising, but as time went on this was reduced by a systematic programme of diagnosis and therapy based on the results of antibiotic sensitivity tests. It seems evident that the high rate of infection in 1970 was due to the fact that gynaecologists underestimated gonorrhoea as the probable cause of their patients' symptoms. They therefore rarely took cultures on special media and even then the cultures were limited to a single specimen. The present study shows that this procedure is inadequate.

In our last two groups the percentage of new cases of gonorrhoea became stabilized at about 12 per cent.; this suggests that a further reduction is unlikely.

In chronic conditions of the genito-urinary tract it is imperative to bear in mind the possibility of a gonococcal infection and to take cultures or use the FAT. According to our results, in the course of 4 years, 251 women in this district of Prague would have failed to be registered as cases of gonorrhoea and would have become the source of further infection.

On the strength of a positive FAT repeated cultures were made and thus a further 94 positive cases were diagnosed. The FAT is not only very useful for the detection of latent infection, but is also less expensive than traditional cultures and provides more rapid results.

\section{References}

Brown, W. (1968) Antibiotic News, 5, 1

Danielsson, D. (1965) Acta derm.-venereol. (Stockh.), 45, 74

Deacon, W. E., Peacock, W. L., Freeman, E. M., and Harris, A. (1959) Proc. Soc. exp. Biol. (N.Y.), 101, 322

Enfors, W., Eriksson, G., KaAman, T., and Krogh, G. voN (1973) Brit. F. vener. Dis., 49, 500

FRY, C. S., and WILKINSON, A. E. (1964) Ibid., 40, 125

Gallwey, J. M. D., Nicol, C. S., and Ridley, $M$. (1967) Ibid., 43, 168

Henderson, R. A., Rutherford, S., Phelps, J. A., and ROBERTSON, P. (1970) Ibid., 46, 205

Kellogg, D. S., and Deacon, W. E. (1964) Proc. Soc. exp. Biol. $(N . \cdot Y$.), 115, 963

Klanica, J., and Stejskalová, M. (1972) Prakt. lék., 52, 261

LIND, I. (1967) Acta path. microbiol. scand., 70, 613

- and Rhodeus, J. M. (1970) Ibid., 78, 153

LuCAS, J. B., Price, E. V., Thayer, J. D., and Schroeter, A. (1967) New Engl. F. Med., 276, 1454

Palouš, V., Šturma, J., Ǩ̆kČEK, J., Potužní, V., and HAUSNER, O. (1971) Cs. Derm., 46, 256

Sommervilie, R. G. (1968) Bull. Wld Hith Org., 39, 942

Thin, R. N. (1970) Brit. F. vener. Dis., 46, 27

, Williams, I. A., and NICOL, C. S. (1971) Ibid., 47, 27 